\title{
Numerical Investigation of Transitional Flow over a Flat Plate under Constant Heat Fluxes
}

\author{
*Gokhan Canbolat, Alperen Yildizeli, Haluk Anil Kose and Sertac Cadirci \\ Department of Mechanical Engineering, Istanbul Technical University, 34437 Istanbul, Turkey
}

\begin{abstract}
In this study, a boundary layer flow over a flat plate is investigated numerically at constant inlet freestream velocity and turbulence intensity. After intensive mesh refinements, an adequate computational domain is determined. Four turbulence models (k-epsilon, k-omega, k-omega SST, Transition SST) are used to analyze the boundary layer flow. Local surface friction coefficient distribution is obtained and compared to each other to assess the most convenient turbulence model. The Computational Fluid Dynamics (CFD) results show that the Transition SST turbulence model demonstrates the most realistic skin friction coefficient $\left(\mathrm{C}_{\mathrm{f}}\right)$ distribution in agreement with the experimental data. Additionally; the effects of constant heat fluxes on $\mathrm{C}_{\mathrm{f}}$ values are investigated and it is found that transition can be pulled back by a heated plate compared to isothermal case. Moreover, it is fount that $\mathrm{C}_{\mathrm{f}}$ values in the turbulent region decrease compared to isothermal case.
\end{abstract}

Key words: Turbulence Models, Active Flow Control, Boundary Layer Flow, Skin Friction Coefficient

\section{Introduction}

In many engineering applications, flow-related problems such as friction, noise and vibration are frequently encountered in external flows around objects. Therefore, the elimination of such flowrelated problems and the reduction of adverse effects of flows are necessary [1].

Due to the intense eddy vortices, local surface friction coefficient is higher in turbulent flow than laminar flow [2]. Through the active flow control methods, transitional flow over a flat plate can be manipulated by heating and cooling which affects viscosity and density of fluid. There are studies in the literature related to active flow control by heating process to manipulate transitional regime and decrease the skin friction coefficient.

Dovgal et al. performed experimental studies in order to obtain transition delay by local heating and showed that local heating can be used for the laminarization of the boundary layer [3]. Schmid and Selberg modelled two dimensional boundary layer flow over a flat plate with and without flush mounted heating strips. The effects of constant wall temperature, increasing and decreasing wall temperatures were studied for different surface heating lengths. They concluded that this technique might be useful in modifying transition and separation locations over airfoils [4]. Jamal investigated the effect of heating strips on transitional flow over a flat plate. The effects of the heat-transfer-strip location, the heating level, the length of the heat-transfer strip, the flow free-stream Mach number, and an isolated roughness element on single-frequency and wideband transition locations in subsonic two-dimensional flow are evaluated. [5]. Kramer et al.,

*Corresponding author: Gokhan Canbolat Address: Department of Mechanical Engineering, Istanbul Technical University, 34437, Istanbul, Turkey. E-mail address: canbolat17@itu.edu.tr, Phone: +902122931300 
investigated drag reduction around existing aircraft configuration and found that heating of the surface reduces turbulent skin friction coefficient [6]. Filippov investigated the influence of plate nose heating of a thermally insulated plate on the development of plate boundary layer experimentally and concluded that heating made it possible to delay laminar-turbulent transition of the boundary layer considerably at comparatively low Reynolds numbers [7]. Subasi and Gunes studied the effect of local and total heating of a flat plate on skin friction coefficient and found that local heating postponed the transition to turbulent flow and all heating process decreased the skin friction coefficient significantly in turbulent region [8].

In this study, a boundary layer flow over a flat plate is investigated numerically at constant inlet freestream velocity and turbulence intensity. The aim of this study is to compare the skin friction coefficient distributions for with and without active flow control by heating plate with constant heat fluxes.

\section{Methodology}

In this section, the methodology of this study is presented. ANSYS-Fluent flow solver is used to perform computational fluid dynamics calculations. Geometry of computational domain, mesh, mesh independence test, boundary conditions and validation with experimental data are given in this section.

\subsection{Geometry}

The computational domain of interest is shown in Figure 1. that is a thin and flat plate with $2 \times 0.8$ $\mathrm{m}^{2}$ surface area. The validity of the constant freestream velocity provided in the entrance area is ensured by leaving a space of $0.15 \mathrm{~m}$ before the start of the plate. The height of the computational domain is chosen $0.3 \mathrm{~m}$ to create a sufficient flow domain to prevent the boundary layer flow from being affected by boundary conditions.

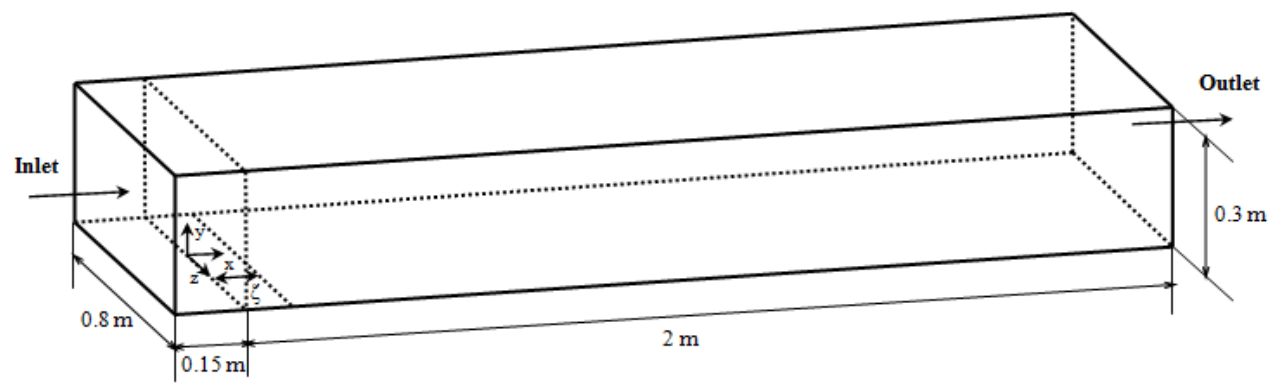

Figure 1. Computational domain

In this case, flow can be assumed to be two dimensional. In order to reduce the computational cost, geometry is modeled as two dimensional and it is validated that $\mathrm{C}_{\mathrm{f}}$ values obtained from numerical calculations are compatible with the 2-D and 3-D numerical results. 


\subsection{Mesh and Mesh Independency Test}

Structured mesh method is chosen to avoid skewness restrictions. Since edges of cells are perpendicular to each other, computation period is highly decreased with selected mesh method. In order to predict the boundary layer flow correctly in streamwise direction, the mesh refinement is done in the vicinity of the flat plate and wall $\mathrm{y}^{+}$values are selected around 1 to be compatible with mesh independency. After intensive mesh independence tests, 161250 rectangular elements are used for the 2-D analyses, and each elements' size in $\mathrm{x}$-direction is $2 \mathrm{~cm}$ as shown in Figure 2.

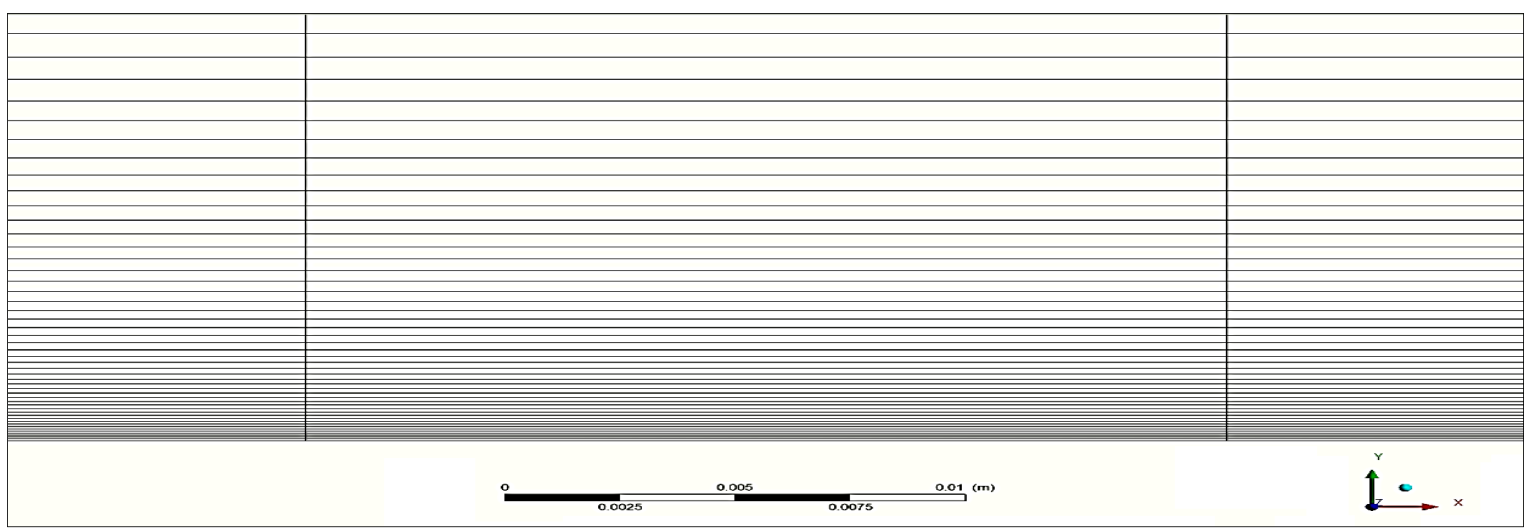

Figure 2. Detailed view of the mesh of computational domain

Mesh independency tests are carried out considering the effect of $\mathrm{y}^{+}$and the mean relative error in $\mathrm{C}_{\mathrm{f}}$ with increasing number of elements. Figure 3. shows how mesh independency tests are done and reveals that a mesh consisting of 161250 elements with $\mathrm{y}^{+}$lower that 1 is adequate to perform CFD analysis.

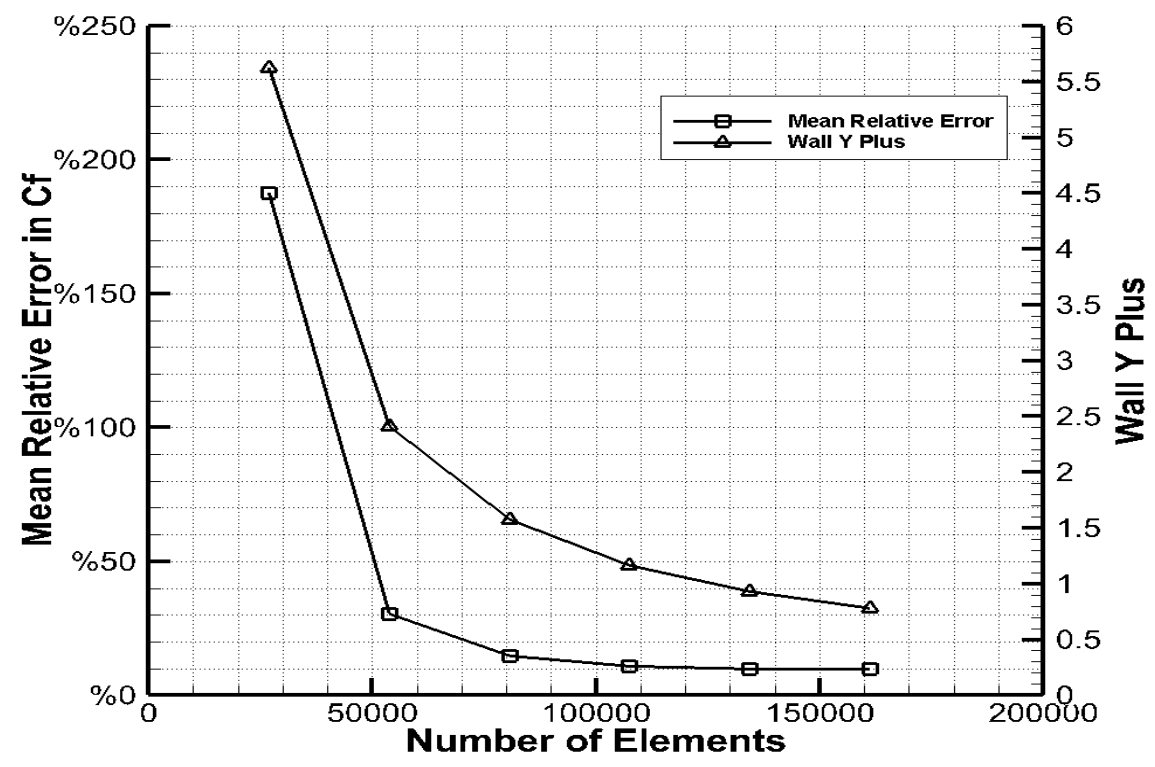

Figure 3. Mesh independency tests 


\subsection{Boundary Conditions}

As inlet boundary condition, uniform freestream velocity and turbulence intensity are specified $19.8 \mathrm{~m} / \mathrm{s}$ and $0.9 \%$ respectively. For outlet boundary condition, outlet gauge pressure is given as atmospheric pressure. The lower wall (flat plate) is stationary with no-slip boundary condition where the remaining control surfaces are specified as moving wall with inlet freestream velocity.

The thermophysical properties of air for the isothermal cases are tabulated in Table 1.

Table 1. Thermophysical Properties of Air

\begin{tabular}{ccccc}
\hline Fluid & Density & Dynamic & Thermal \\
& {$[\mathrm{kg} / \mathrm{m} 3]$} & $\begin{array}{c}\text { Viscosity } \\
{[\mathrm{kg} / \mathrm{ms}]}\end{array}$ & $\begin{array}{c}\text { Specific Heat } \\
{[\mathrm{J} / \mathrm{kgK}]}\end{array}$ \\
\hline Air & 1.125 & $1.7894 \times 10^{-5}$ & 0.0242 & 1006.43 \\
\hline
\end{tabular}

In this study, constant heat flux is also implemented in order to investigate the changes in $\mathrm{C}_{\mathrm{f}, \mathrm{x}}$ effects. Since the viscosity and density are effected by temperature ideal gas assumption and the Sutherland Law (Eq. 1) are used in further CFD calculations.

$\mu=\frac{C T^{3 / 2}}{T+S}$

In Sutherland Law, $C$ and $\mathrm{S}$ are constants for air which are $1.458 \times 10^{-6}\left[\mathrm{~kg} / \mathrm{ms} \mathrm{K}^{1 / 2}\right]$ and $110[\mathrm{~K}]$ respectively.

\subsection{Governing Equations}

The governing equations used in the CFD modelling are continuity, Navier-Stokes and energy equations for steady, incompressible, 2D flows given in Equations (2a), (2b) and (2c) respectively.

$$
\begin{aligned}
& \frac{\partial \rho}{\partial \mathrm{t}}+\frac{\partial}{\partial \mathrm{x}_{\mathrm{i}}}\left(\rho \mathrm{u}_{\mathrm{i}}\right)=0 \\
& \frac{\partial\left(\rho \mathrm{u}_{\mathrm{i}}\right)}{\partial \mathrm{t}}+\frac{\partial}{\partial \mathrm{x}_{\mathrm{j}}}\left(\rho \mathrm{u}_{\mathrm{i}} \mathrm{u}_{\mathrm{j}}\right)=-\frac{\partial \mathrm{p}}{\partial \mathrm{x}_{\mathrm{i}}}+\frac{\partial}{\partial \mathrm{x}_{\mathrm{j}}}\left[\mu\left(\frac{\partial \mathrm{u}_{\mathrm{i}}}{\partial \mathrm{x}_{\mathrm{j}}}+\frac{\partial \mathrm{u}_{\mathrm{j}}}{\partial \mathrm{x}_{\mathrm{i}}}-\frac{2}{3} \delta_{\mathrm{ij}} \frac{\partial \mathrm{u}_{1}}{\partial \mathrm{x}_{1}}\right)\right]+\frac{\partial}{\partial \mathrm{x}_{\mathrm{j}}}\left(-\rho \overline{\mathrm{u}_{\mathrm{i}}{ }^{\prime} \mathrm{u}_{\mathrm{j}}{ }^{\prime}}\right) \\
& \frac{\partial(\rho \mathrm{E})}{\partial \mathrm{t}}+\frac{\partial}{\partial \mathrm{x}_{\mathrm{j}}}\left[\mathrm{u}_{\mathrm{i}}(\rho \mathrm{E}+\mathrm{p})\right]=\frac{\partial}{\partial \mathrm{x}_{\mathrm{j}}}\left[\left(\mathrm{k}+\frac{\mathrm{c}_{\mathrm{p}} \mu_{\mathrm{t}}}{\operatorname{Pr}_{\mathrm{t}}}\right) \frac{\partial \mathrm{T}}{\partial \mathrm{x}_{\mathrm{j}}}+\mathrm{u}_{\mathrm{i}}\left(\tau_{\mathrm{i}}\right)_{\mathrm{eff}}\right]+\delta_{\mathrm{h}}
\end{aligned}
$$


In addition to the governing equations, the transport equations for the Reynolds Averaged Navier-Stokes (RANS) turbulence models are used by the flow solver. Since transitional flow can be modelled correctly by SST-Transition (Shear Stress Transport Transition), the transport equation for the intermittency $\gamma$ is given in Equations (3). The transition sources in Equation (3) can be obtained from [9].

$$
\left.\frac{\partial(\rho \gamma)}{\partial t}+\frac{\partial\left(\rho U_{J} \gamma\right)}{\partial x_{j}}=P_{\gamma 1}-E_{\gamma 1}+P_{\gamma 2}-E_{\gamma 2}+\frac{\partial}{\partial x_{j}}\left[\left(\mu+\frac{\mu_{t}}{\sigma_{\gamma}}\right) \frac{\partial \gamma}{\partial x_{j}}\right)\right]
$$

For a parallel flow over a flat plate the aerodynamic drag forces are only caused by frictional forces which is defined in the non-dimensional form of the wall shear stress that is skin friction coefficient $\mathrm{C}_{\mathrm{f}}$. In our study, distribution of skin friction coefficient in stream wise direction is assumed to be an important indicator to test the validity of turbulence model.

Further, shape factor $(\mathrm{H})$ is also calculated and for the base flow with the isothermal boundary conditions and compared to the experimental data. Shape factor is a non-dimensional number which indicates the shape of the velocity profile. Shape factor can be integrated numerically from the ratio of the displacement thickness to the momentum thickness as shown in Eq. (4). In Equation (4), $\delta^{*}$ is displacement thickness, $\theta$ is the momentum thickness and $U(y)$ is the velocity profile.

$$
H=\frac{\delta^{*}}{\theta}=\frac{\int_{0}^{\infty}\left(1-\frac{U(y)}{U_{\infty}}\right) d y}{\int_{0}^{\infty} \frac{U(y)}{U_{\infty}}\left(1-\frac{U(y)}{U_{\infty}}\right) d y}
$$

\section{Results}

\subsection{Validation and Verification of the Base Flow}

In the first stage of the study, available experimental data [10] is used for the validation of the turbulence model based on local skin friction coefficient as shown in Figure 4. In addition to the experimental data, empirical correlations of $\mathrm{C}_{\mathrm{f}}$ for the laminar and turbulent regimes are used in the comparison of the turbulence models. The $\mathrm{C}_{\mathrm{f}}$ correlations for the laminar and turbulent regimes are given in Equations (5).

$$
\mathrm{C}_{\mathrm{f}, \mathrm{lam}}=\frac{0.664}{\sqrt{\mathrm{Re}_{\mathrm{x}}}} \quad \mathrm{C}_{\mathrm{f}, \text { turb }}=\frac{0.455}{\ln ^{2}\left(0.06 \mathrm{Re}_{\mathrm{x}}\right)}
$$




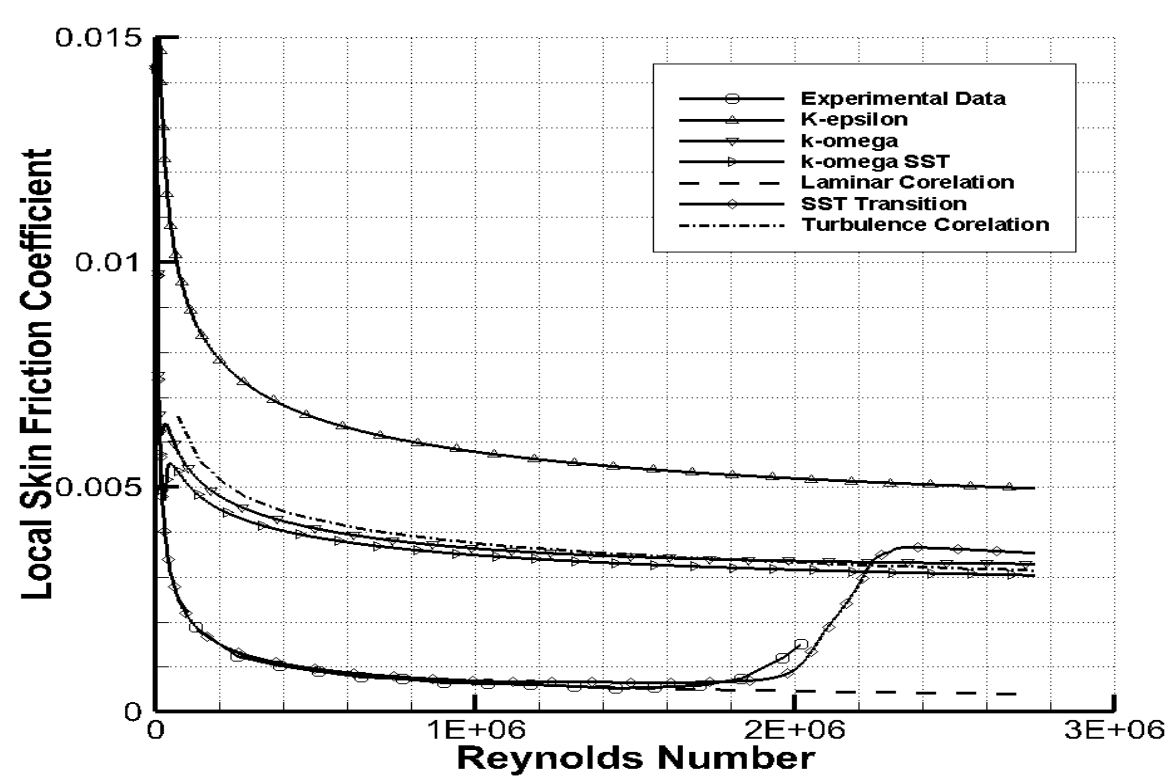

Figure 4. Validation and verification of turbulence models

As shown in Figure 4, SST-Transition model represents the most accurate distribution of local skin friction coefficient which fits to both experimental and empirical data. Thus, this turbulence model is selected for further calculations and predicting the location of transition in active flow control.

Figure 5. shows the comparisons of the velocity profiles obtained from CFD calculations using SST-Transition model and experimental data in [10] at $\mathrm{x}=0.795 \mathrm{~m}$ and $\mathrm{x}=1.495 \mathrm{~m}$. The comparisons show a perfect agreement between numerical and experimental data.
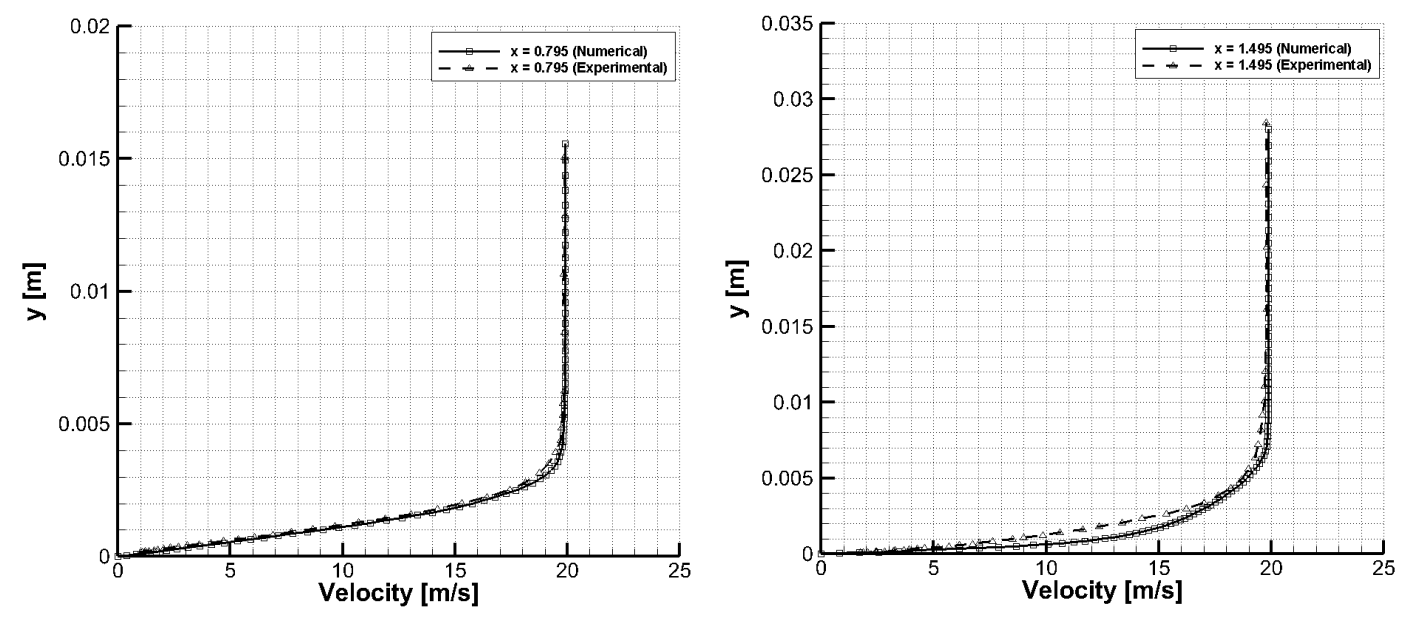

Figure 5. Comparisons of velocity profile at $x=0.795 \mathrm{~m}$ and $\mathrm{x}=1.495 \mathrm{~m}$ 
According to Figure 6., experimental in [10] and numerical results of shape factor are compared and they agree with each other satisfactorily. The mean relative error between experimental and CFD result is found $4.58 \%$.

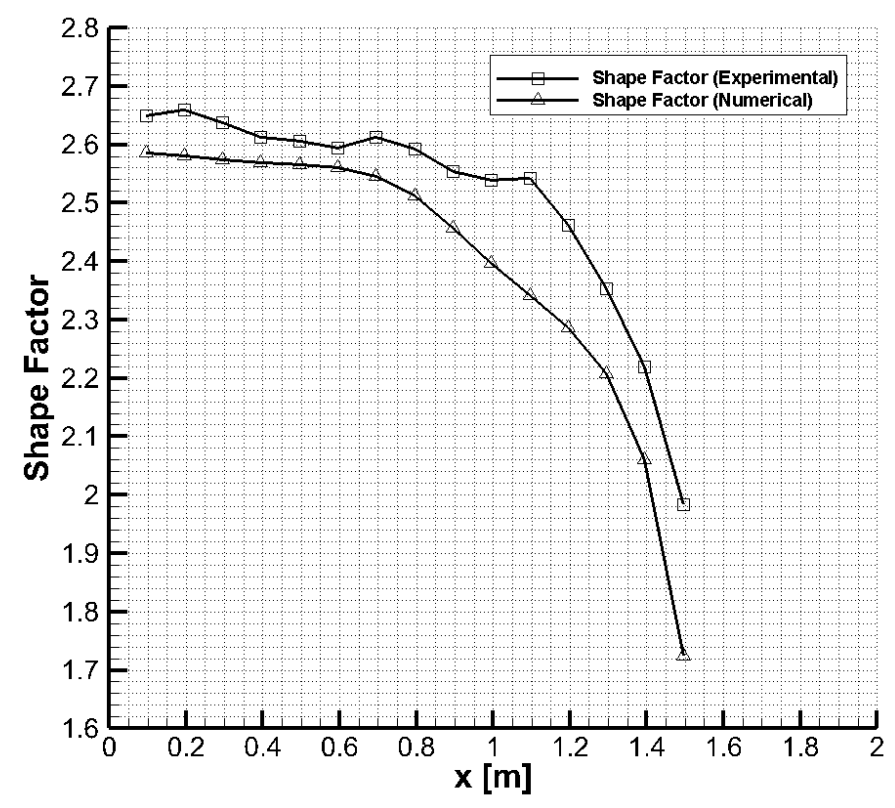

Figure 6. Comparison of shape factor along the flat plate

\subsection{Active Flow Control for the Manipulation of Transition}

Active flow control methods include either momentum or energy transfer in order to manipulate the base flow to achieve certain objective. In our study, we performed heating of the flat plate as an active flow control method to pull transition region back. In this respect the flat plate is heated completely at different constant heat fluxes for freestream turbulence intensities $1 \%, 3 \%$ and $8 \%$ as displayed in Figure (7a), (7b) and (7c) respectively.

In general, the viscosity of a gas increases with temperature. For liquids, transition delay can be obtained with a surface that is hotter that the liquid freestream. For gases, transition delay can be obtained with a surface that is colder than the gaseous freestream [11] as indicated in Figure (7d). In our study, transition control is performed by the heated flat plate that caused transition being pulled back. It is observed that with increasing heat flux, critical Reynolds number and the overall skin friction coefficient in the turbulent regime are decreased. It should be mentioned that the total gain from this active control also depends on the length of the flat plate.

Another factor which effects is the freestream turbulence intensity. The effect of the heating remains the same but with increasing freestream turbulence intensity the flow is more likely to become turbulent. 

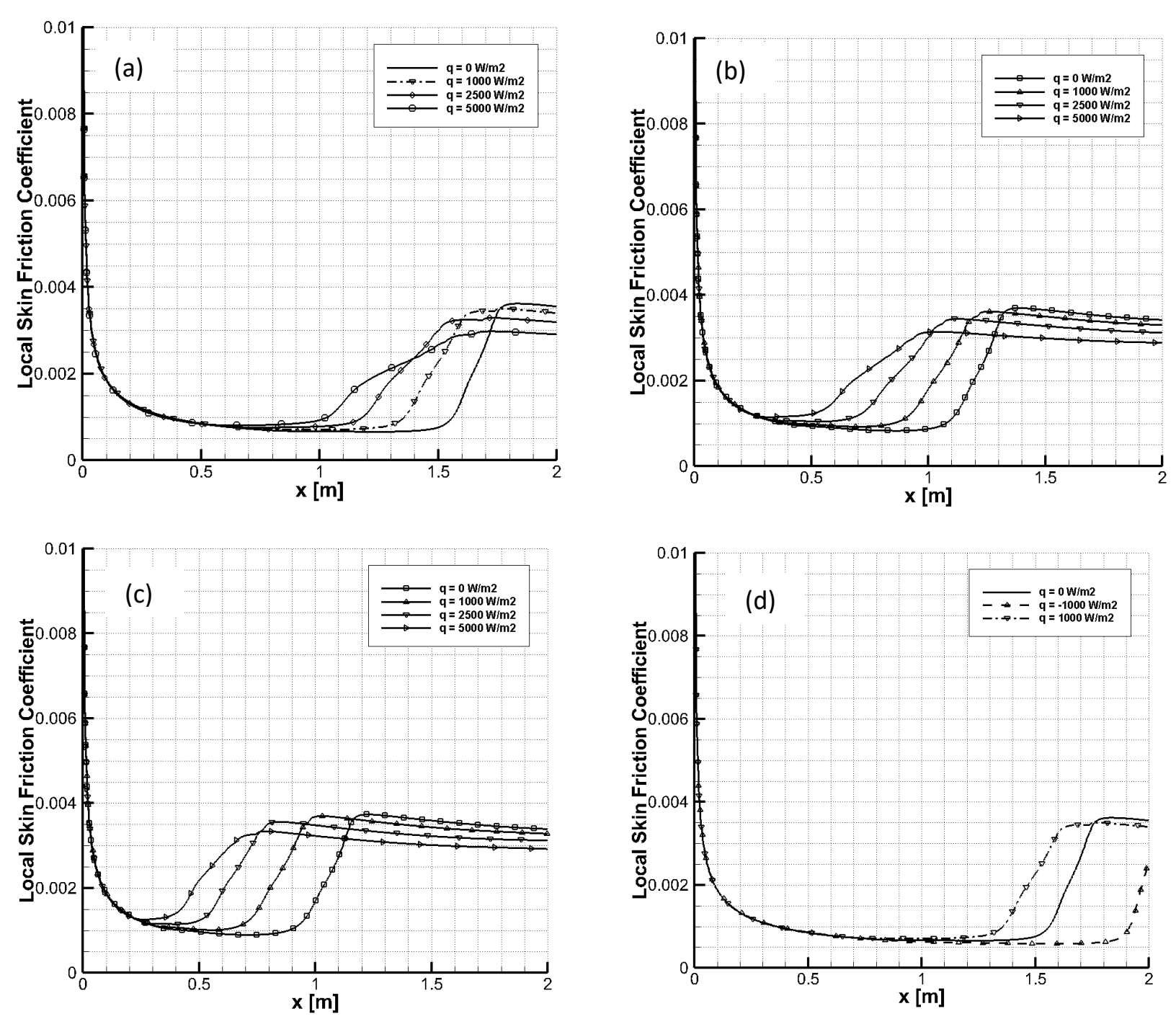

Figure 7. Effect of heat fluxes on transition for (a) $1 \%$, (b) $3 \%$ and (c) $8 \%$ freestream turbulence intensities; and the (d) comparison of heating, cooling

\section{Conclusions}

In this study, transition control over a flat plate is investigated numerically. SST-Transition model is found to be the best assessment for CFD calculations since it can predict transition region at the critical Reynolds number. A completely uniform heated flat plate can manipulate transitional regime and pulls it closer to the leading edge. It is found that the flow becomes more likely to be turbulent if the heat flux and the freestream turbulence intensity are increased. It is proved numerically that heating or cooling of the surface is an effective active flow control method to manipulate transition. It is concluded that a heated flat plate induces the decrease of the local skin friction coefficient in the turbulent region for an incompressible gaseous flow. 


\section{References}

[1] Akansu Y.E., Ozmert M. and Firat E., 2011, The Effect of Attact Angle to Vortex Shedding Phemenon of Flow Around a Square Prism With a Flow Control Rod, J. of Thermal Science and Technology, 31, 1, 109-120.

[2] Cengel Y.A. and Cimbala J.M., Fluid Mechanics: Fundamentals and applications, 2008.

[3] Dovgal A.V., Levchenko V.Y.A. ve Timofeev V.A., 1989, Boundary Layer Control by a Local Heating of the Wall, IUTAM Symposium on Laminar-Turbulent Transition, Toulouse, France.

[4] Schmid, S. and Selberg, B., 1992, Analysis of the Effect of Heat Strips on Boundary Layer Development Over a Flat Plate, SAE Technical Paper 921923.

[5] Jamal A. Masad, Transition in flow over heat-transfer strips, Physics of Fluids 7, 2163 (1995); doi: 10.1063/1.868466.

[6] Kramer B.R., Smith B.C., Heid J.P., Noffz G.K., Richwine D.M. ve Ng T., 1999, Drag Reduction Experiments Using Boundary Layer Heating, 37th AIAA Aerospace Sciences Meeting \& Exhibit, Reno, NV.

[7] Filippov V.M., 2002, Influence of Plate Nose Heating on Boundary Layer Development, Fluid Dynamics, 37/1, 27- 36.

[8] Subas1 A., Gunes H., Effect of wall heating on boundary layer transition over a flat plate, J. of Thermal Science and Technology, 2015.

[9] ANSYS Fluent, Theory’s Guide, November 2013, Release 15.0.

[10] ERCOFTAC (European Research Community on Flow, Turbulence and Combustion) Nexus. [Online database], URL: http://www.ercoftac.org [15 August 2018].

[11] Gad el Hak M., Flow Control: Active, Passive and Reactive Flow Management, Cambridge University Press, 2000. 